\title{
Quality of medical care for people with and without comorbid mental illness and substance misuse: systematic review of comparative studies
}

\author{
Alex J. Mitchell, Darren Malone and Caroline Carney Doebbeling
}

\section{Background}

There has been long-standing concern about the quality of medical care offered to people with mental illness.

\section{Aims}

To investigate whether the quality of medical care received by people with mental health conditions, including substance misuse, differs from the care received by people who have no comparable mental disorder.

\section{Method}

A systematic review of studies that examined the quality of medical care in those with and without mental illness was conducted using robust critical appraisal techniques.

\section{Results}

Of 31 valid studies, 27 examined receipt of medical care in those with and without mental illness and 10 examined medical care in those with and without substance use disorder (or dual diagnosis). Nineteen of 27 and 10 of 10, respectively, suggested inferior quality of care in at least one domain. Twelve studies found no appreciable differences in care or failed to detect a difference in at least one key area. Several studies showed an increase in healthcare utilisation but without any increase in quality. Three studies found superior care for individuals with mental illness in specific subdomains. There was inadequate information concerning patient satisfaction and structural differences in healthcare delivery. There was also inadequate separation of delivery of care from uptake in care on which to base causal explanations.

\section{Conclusions}

Despite similar or more frequent medical contacts, there are often disparities in the physical healthcare delivered to those with psychiatric illness although the magnitude of this effect varies considerably.

\section{Declaration of interest}

None.
Medical comorbidity in those with established mental illness is an area of great concern. ${ }^{1,2}$ The combination of medical and psychiatric illness has been linked with increased functional and occupational disability, ${ }^{3-6}$ poorer quality of life $^{7-9}$ and accelerated mortality ${ }^{10}$ compared with those with one diagnosis alone. Medical illness affects more than half of those with mental illness, particularly in older people. ${ }^{1-15}$ Similarly the overlap of substance misuse and medical burden is well recognised. ${ }^{16-19}$ Given this clinically significant overlap, it is important to ask whether this comorbidity is appropriately recognised and treated in routine practice. National guidance in the UK and the USA highlights that the medical care of people with mental disorders is paramount. ${ }^{20,21}$ Once a mental health problem is recognised adequate care depends on the uptake and delivery of appropriate treatment. ${ }^{22}$ It is not yet clear what proportion of physical health needs and treatment are unmet in this population compared with individuals without documented mental illness. Although one uncontrolled study recently reported that among the homeless mentally ill $43.6 \%$ had an unmet need for medical care, overall little is known about other populations of people with mental illness. ${ }^{23}$

In this systematic review, we looked at whether the quality of medical care received by people with mental illness is different from the care received by those who have no comparable mental disorder. By using only studies that recruited comparative data we wished to ascertain whether inequalities in care existed as a result of psychiatric diagnoses (or closely affiliated factors).

\section{Method of systematic review}

For this study we used the definition of quality of care provided by the Institute of Medicine and endorsed by the National Quality
Measures Clearinghouse (NQMC), part of the US Department of Health and Human Services. ${ }^{24}$ They define quality of care as 'the degree to which health care services for individuals and populations increase the likelihood of desired health outcomes and are consistent with current professional knowledge'. Further, the NQMC suggests five domains of measurement that contribute to healthcare quality. ${ }^{25}$ These are:

(a) access: assesses the patient's attainment of timely and appropriate healthcare;

(b) outcome: a health state of a patient resulting from healthcare;

(c) patient experience: aggregates reports of patients about their observations of and participation in healthcare;

(d) process: healthcare service provided to, or on behalf of, a patient;

(e) structure: a feature of a healthcare organisation or clinician relevant to its capacity to provide healthcare.

\section{Search}

The following abstract databases were searched. MEDLINE 1966 to May 2008, PsycINFO 1887 to May 2008 and EMBASE 1980 to May 2008. In these databases the keywords/MeSH terms ('comorbidity OR comorbidity' OR 'organic OR physical OR medic $^{\star}$ OR diabetes OR cardia* OR neurolog* OR osteoporosis OR arthritis OR cancer OR HIV OR AIDs OR hepatitis OR stroke OR parkinson* OR epilepsy') AND ('psychi* OR mental OR depress* OR schizophr* OR bipolar OR anxiety' OR serious mental illness OR SMI) were used. Six full-text collections were searched: Science Direct, Ingenta Select, SpringerLink, Blackwell 
Synergy, Ovid Full text and Wiley Interscience. In these online databases the same search terms were used but as a full-text search and as a citation search. The abstract database Web of Science/ Web of Knowledge (4.0, ISI) was searched, using the above terms as a text word search, and using key papers in a reverse citation search. Finally, a number of journals were hand searched and several experts contacted. The data extraction was performed in accordance with Quality of Reporting of Meta-analyses guidelines. $^{26}$

\section{Critical appraisal}

Critical appraisal guidelines provided by the Oxford Centre for Evidence-Based Medicine ${ }^{27}$ and criteria suggested by Craig et al were adapted. ${ }^{28}$ Two reviewers examined all the primary studies (A.J.M. and D.M.). Studies were appraised using a 20-point scale in six categories: study design, quality of the sample under study, adequacy of comparators, adequacy of consideration of possible confounding factors, adequacy of the outcome measure(s) and the degree to which a study attempted to delineate delivery from uptake of care (see Appendix for further details). In addition, we assessed the setting of each study, the type and severity of mental illness under study and the length of follow-up (where applicable). In three cases where there was disagreement concerning methodological quality, the lowest value was chosen.

\section{Results}

Our preliminary search identified 428 references (Fig.1). Of these, 72 were primary data studies but only 40 specifically compared the quality of care indices in individuals with and without mental health problems. These were reviewed in detail and nine studies were excluded because they did not examine a medical comorbidity comparison group and/or they examined an outcome that did not fulfil the definition of quality of care above. We excluded studies examining receipt of screening or preventive services in individuals with and without mental health problems because this population had no proven medical illness at the time of study. ${ }^{29-34}$ We also excluded those studies that looked at physical health or mortality but not quality of care. ${ }^{35}$ We excluded studies that examined physical health markers but without assessment of quality of care/service delivery (such as those looking purely at glycosylated haemoglobin $\left(\mathrm{HbA}_{1 \mathrm{c}}\right)$ control in diabetes). ${ }^{36-39}$ We

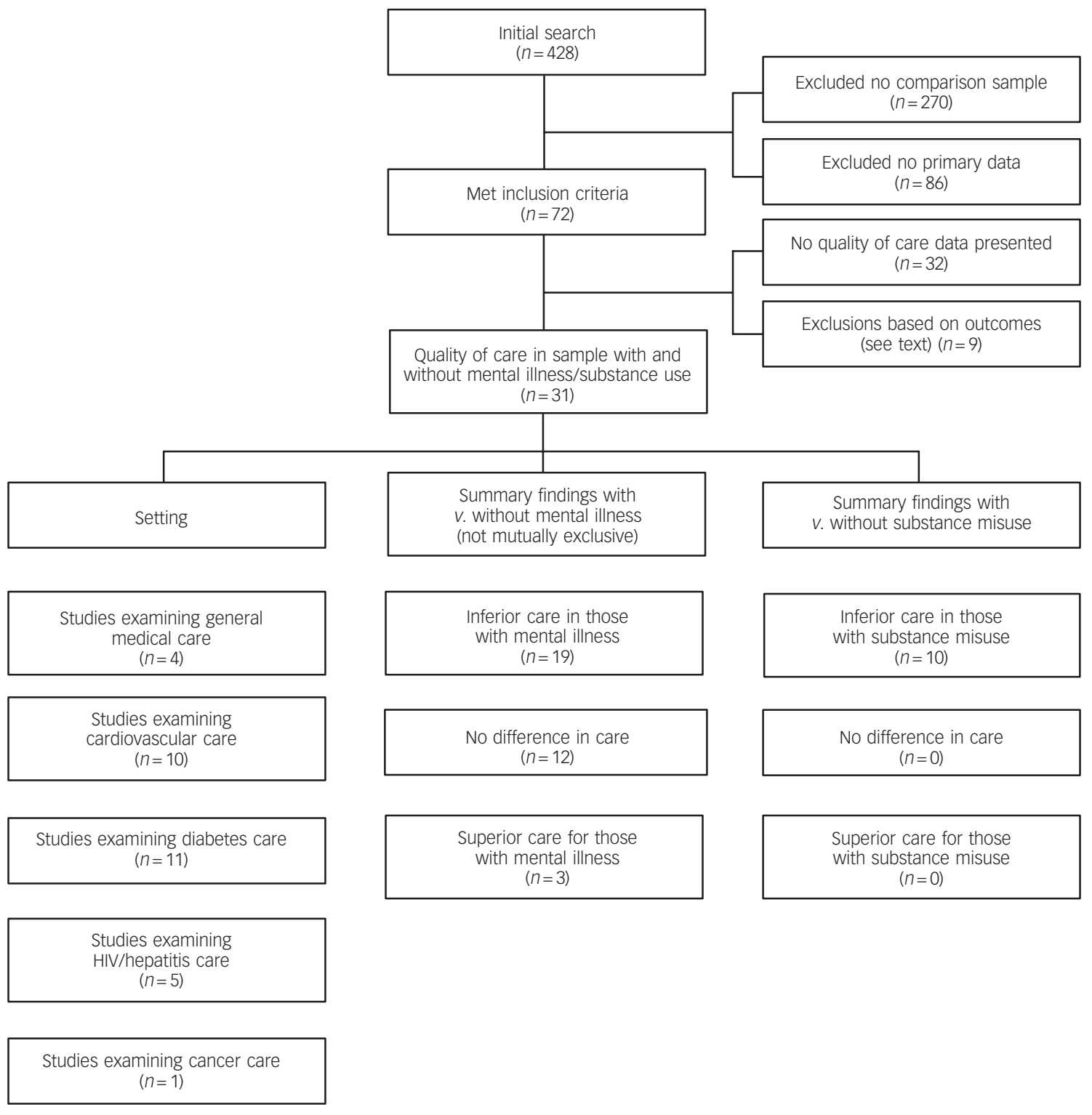

Fig. 1 QUOROM (Quality of Reporting Meta-Analyses) flow diagram 
excluded studies examining adherence, medical adverse events and medical errors. ${ }^{40}$ We also excluded studies that looked at care exclusively in those with non-comorbid mental illness and those studies that lacked a clear comparison sample, ${ }^{41-44}$ and those that measured symptoms of mental illness without defining a disorder. ${ }^{45}$ However, we allowed studies that offered a comparison with population data or data from previous work. This formed one of the critical appraisal criteria.

After these inclusion and exclusion criteria were applied, we found 31 studies that examined individuals with known medical illness and psychiatric illness (online Table DS1). These reported on the quality of general medical care $(n=4),{ }^{46-49}$ cardiovascular care $(n=10),{ }^{50-59}$ diabetes care $(n=11),{ }^{60-70}$ hepatitis/HIV care $(n=5)^{71-75}$ and cancer care $(n=1){ }^{76}$ Three reports reported different aspects of a single sample. ${ }^{62,66,69}$

All studies examined people with mental illness, but differed in their definition of psychiatric disorder. Ten studies examined the quality of care in those known to have harmful substance misuse habits. ${ }^{47,48,50-52,59,60,63,73,74}$ Almost all studies examined process measures (care offered), access (healthcare utilisation) and to a lesser extent outcome (morbidity and mortality) according to the NQMC definition of quality of care. Few examined patient satisfaction or structural aspects of care delivery.

\section{Quality of general (internal) medicine care}

Four studies looked at the quality of care in general (internal) medicine, most employing large medical databases to access retrospective data on quality of care and healthcare attendance (utilisation) (online Table DS2). Redelmeier and colleagues looked at over one million older individuals as part of the Ontario drug benefit programme. ${ }^{46}$ Individuals with psychotic illness were significantly less likely to have received treatment for their unrelated comorbid illnesses. This was also true for individuals with diabetes and pulmonary emphysema. A weakness of this study is that those with psychotic illness were identified by prescriptions for haloperidol alone.

Desai et al utilised a Veterans Health Administration database to identify 90240 people with a diagnosis of obesity and/or hypertension. ${ }^{47}$ Individuals with dual diagnosis (mental illness and substance use disorders) were significantly less likely to have received health promotion counselling although the overall magnitude of the difference was small (2\%). There was no difference with regard to people with mental illness alone. Cradock-O'Leary et al used a large Veterans Health Administration database to compare those with a primary diagnosis of mental illness $(n=47516)$ with those without, both by use of medical services and by the number of medical visits. ${ }^{48}$ The authors found that those with mental illness (including those with substance misuse) had the lowest rates for the number of medical visits. Individuals older than 50 years were least likely to have received medical care if they had a diagnosis of substance misuse, depression, bipolar disorder, anxiety disorder or post-traumatic disorder, after partial control for healthcare utilisation by excluding non-attenders. In a small study, Dickerson and colleagues recruited 100 people with schizophrenia and 100 with a mood disorder from psychiatric out-patient clinics in Baltimore. ${ }^{49}$ Self-reported rates of healthcare were compared with population norms who were matched by age and ethnicity and who were physically healthy. Compared with those, the participants with mental illness visited a doctor and received a physical examination more often. However, those with mental illness reported more perceived barriers to receiving medical care and were less likely to receive routine dental care. This study could have been improved with controlling for number and/or severity of medical diagnoses.

\section{Quality of cardiovascular care}

Ten studies examined the comparative quality of cardiovascular care in people with and without a mental illness. In a large retrospective national Medicare cohort study of 113653 hospitalised people aged 65 years or older, Druss and colleagues (2000) examined cardiovascular care following an acute myocardial infarction. ${ }^{50}$ The $4.7 \%$ with a comorbid mental disorder were compared with those who did not have a 'current and ongoing' psychiatric illness. Individuals with comorbidity were significantly less likely to undergo percutaneous transluminal coronary angiography or coronary artery bypass graft surgery. After adjusting for demographic, clinical, hospital and regional factors, those with mental disorders were only $41 \%$ (for schizophrenia) to $78 \%$ (for substance misuse) as likely to undergo cardiac catheterisation as those without mental disorders. In 2001 Druss and colleagues repeated the methodology of their previous study, identifying individuals hospitalised for myocardial infarction of whom 4664 had a secondary diagnosis of mental disorder. ${ }^{51}$ Compared with those without a psychiatric disorder, people with schizophrenia were less likely to have reperfusion, beta-blockers and angiotensin-converting enzyme inhibitors. People with affective disorders were less likely to have reperfusion and aspirin and those with substance use disorders were less likely to be given angiotensin-converting enzyme inhibitors. Mental disorder of all types was associated with a $19 \%$ increase in mortality at 1 year. Importantly, when the five quality indicators were added to the model the association was no longer significant, suggesting that elevated mortality is related to poor quality of care.

In one of the largest studies to date, Young et al identified 25237 people with mental illness who had suffered a myocardial infarction. ${ }^{52}$ The authors examined rates of revascularisation procedures (cardiac catheterisation, percutaneous transluminal coronary angiography and coronary artery bypass graft surgery) and mortality, stratified by age. Those with a mental illness had significantly lower levels of all three revascularisation procedures compared with those without mental illness, with the lowest rates seen in those older than 64 years. With regard to in-patient mortality post-myocardial infarction, in the older cohort (older than 65 years) with mental illness there was a $21 \%$ lower riskadjusted likelihood of death $(P<0.001)$ compared with those without mental illness. In the younger cohort those with schizophrenia and substance misuse had higher in-patient mortality rates (both $P<0.001$ ).

Desai and colleagues used a national sample of 5886 veterans aged under 65 years discharged from Veterans Administration hospitals with a principal diagnosis of acute myocardial infarction. ${ }^{53}$ After controlling for numerous variables (online Table DS2), quality of care was found to be similar in those with and without mental illness, but individuals with a substance use disorder were significantly less likely to be taking beta-blockers than those without such a disorder. Lawrence et al used Western Australia linked databases to identify 210129 users of mental health services with an ICD-9 diagnosis of mental illness (including diagnoses of dementia). ${ }^{54}$ Men with schizophrenia were only $60 \%$ as likely to be admitted for ischaemic heart disease as those in the general population (despite being 1.8 times more likely to die from ischaemic heart disease). People with dementia also had lower rates of admission for ischaemic heart disease. Revascularisation rates were lowest in dementia, followed by schizophrenia, substance misuse, other psychoses and affective psychosis (rate ratios $0.14,0.31,0.60,0.66,0.77$ respectively). This 
is one of the few studies to examine the quality of care in people with dementia as well as other mental illnesses.

Petersen et al (2003) examined the records of 4340 male veterans discharged after a clinically confirmed myocardial infarction. ${ }^{55}$ The authors identified mental disorder using admission to a psychiatric hospital, attendance at a psychiatric or drug/alcohol clinic or lifetime mental health diagnosis. Using these definitions, the only significant difference was that those with mental illness were less likely to undergo in-patient diagnostic angiography. There was no statistical difference in the uptake of coronary artery bypass graft surgery, receipt of physical health medications or mortality at 1 year. This method of identifying mental disorder was similar to that employed by Jones and colleagues. ${ }^{56}$ The authors compared the receipt of percutaneous transluminal coronary angiography and coronary artery bypass graft surgery within 30 days of discharge after hospitalisation for an acute myocardial infarction by those with and without mental disorder but found no significant difference in the rates of revascularisation.

Wang's group looked at antihypertensive prescriptions and the influence of comorbid chronic medical conditions, including depression. ${ }^{57}$ After controlling for comorbidity and healthcare utilisation, individuals with depression were significantly less likely to receive antihypertensives than those with asthma and chronic obstructive pulmonary disease. Hippisley-Cox and colleagues used the QRESEARCH primary care database to identify 127932 people with coronary heart disease, of whom 701 had a diagnosis of schizophrenia or bipolar disorder. ${ }^{58}$ The authors used quality of care indicators defined in the UK General Medical Services contract. Compared with individuals without mental disorder, there were no differences in the offer of smoking cessation advice or prescription of aspirin, antiplatelets, anticoagulants or beta-blockers, but patients with schizophrenia were $15 \%$ less likely to have a recent prescription for a statin and $7 \%$ less likely to have cholesterol levels recorded. No significant differences were apparent in those with bipolar disorders. Li et al examined the New York State hospital discharge data and Cardiac Surgery Report of Surgeon's individual mortality rate. ${ }^{59}$ After controlling for sociodemographic variables people with mental disorders (and those with both substance use disorder and psychiatric disorder) were significantly more likely to be treated by low-quality surgeons for coronary artery bypass graft surgery.

\section{Quality of diabetes care}

Of eleven studies that compared the quality of diabetes care in individuals with and without mental illness only one group did not use a large insurance or clinical database and this group published results in three publications. ${ }^{62,66,69}$ Desai and colleagues examined the Veterans Health Administration's database in the USA and identified 38020 individuals with a diagnosis of diabetes who had had at least three out-patient visits in the previous year. ${ }^{60}$ They found that individuals with substance use disorders were significantly less likely to receive a retinal or foot sensory examination even though those with a mental disorder had significantly more out-patient visits in the previous year. Lin and colleagues ${ }^{61}$ conducted a population survey of 4500 primary care patients with diabetes for the presence of major depression on the Patient Health Questionnaire. A slightly higher proportion of individuals with depression received no $\mathrm{HbA}_{1 \mathrm{c}}$ test in the prior year. Otherwise, there was no difference between individuals with/ without depression in use of diabetes monitoring and preventive services. Jones et al identified 26020 adults from the Blue Cross Blue Shield database, of whom $25 \%$ had a coexisting mental disorder. ${ }^{63}$ Those with mental disorder had higher healthcare utilisation with significantly increased rates of diabetes complications. Nevertheless, after controlling for age, gender, residence, presence of diabetes complications, healthcare utilisation and comorbidity, people with mental illness were significantly less likely to receive $\mathrm{HbA}_{1 \mathrm{c}}$ or cholesterol measurement, with a delay in the receipt of all four diabetic services measured, by an average of 6.6 months $(P<0.001)$.

Frayne et al examined 76799 patients with diabetes and a comorbid mental health condition. ${ }^{64}$ The authors compared those with and without a mental health diagnosis for three indices of diabetes monitoring and two additional outcome measures. Those with a mental health diagnosis were statistically less likely to receive all monitoring, individually and collectively. Krein et al (2006) compared 18273 people with both diabetes and a comorbid serious mental illness with a randomly selected agematched sample that did not have a diagnosis of serious mental illness. ${ }^{65}$ All participants needed to have had at least one primary care or mental healthcare visit during the year to be included in the study; hence there was partial control for healthcare utilisation. During the course of the year under consideration, individuals with both diabetes and serious mental illness had an average of one more visit to primary care and specialist care than people with diabetes alone. Despite this higher attendance there was little quality of care difference.

Sullivan and colleagues looked specifically at rates of hospitalisation for people with diabetes after emergency attendance. ${ }^{66}$ After adjustment for age, gender and ethnicity, those with diabetes and co-occurring mental illness were less likely than those without mental illness to be hospitalised after an emergency department visit. Individuals with diabetes and co-occurring non-psychotic disorders were at especially high risk of not being admitted. Most recently, Weiss and colleagues at the Massachusetts General Hospital compared parameters of care received by 214 people with diabetes and schizophrenia or a schizophrenia-related syndrome and 3594 with diabetes alone. ${ }^{68}$ People with schizophrenia received similar treatment. In fact those with schizophrenia were slightly more likely than the comparison group to receive insulin therapy. Also among individuals with heightened cholesterol levels those with schizophrenia were more likely than the comparison group to be on a lipid-lowering agent and to be referred for nutritional consultation. However, although individuals with hyperlipidaemia in the two groups were equally likely to receive some form of lipid-lowering therapy, those with schizophrenia were twice as likely to receive one of the older, non-statin agents.

In a small clinical survey Dixon et al recruited 100 people with type II diabetes and schizophrenia-spectrum disorder, 101 with diabetes and a major depressive disorder and 99 controls with diabetes from primary care. ${ }^{62}$ Despite similar levels of healthcare utilisation, those with diabetes and schizophrenia received a significantly lower amount of diabetes-related education, resulting in significantly poorer knowledge of their diabetes. Kreyenbuhl et al and Goldberg et al reported further analysis of the Dixon et al study. ${ }^{62,66,69}$ Two hundred and one people with serious mental illness and 99 without serious mental illness from six public and private out-patient mental health clinics $(25 \%$ in the Veterans Health Administration) were compared. Serious mental illness was defined as either a diagnosis of a schizophrenia-spectrum disorder (schizophrenia or schizoaffective disorder) or a major mood disorder (bipolar disorder or recurrent major depressive disorder) as recorded in their medical chart. There were no differences in diabetes outcomes (glucose control, cholesterol or blood pressure levels) but on logistic regression people with diabetes and schizophrenia were only $29 \%$ as likely $(P<0.01)$ and people with diabetes and mood disorders were only $14 \%$ as likely $(P<0.0007)$ 
as those without serious mental illness to receive a statin medication. Additionally, those with serious mental illness received a significantly lower standard of care, diabetes education $(48 \%$ v. $71 \%)$ and written material $(29 \%$ v. $55 \%)$. Frequency of healthcare visits were similar. The only quality of care index in favour of those with mental illness was smoking cessation counselling but this was not adjusted for baseline frequency of smoking. Finally Whyte et al examined quality of care indicators derived from the General Medical Services contract for UK general practitioners. ${ }^{70}$ There was no difference in process measures of care, although unexpectedly individuals with mental illness had better $\mathrm{HbA}_{1 \mathrm{c}}$ control.

\section{Quality of HIV/hepatitis care}

Five studies compared the quality of care for individuals with HIV or related disease. Butt et al examined 354 people with hepatitis C seen in a specialty clinic, of whom 139 had a psychiatric diagnosis. ${ }^{71}$ Only $29.4 \%$ received interferon treatment for 4 weeks or longer but there were no appreciable differences in those with $v$. without psychiatric comorbidity (27.3\% v. 30.7\%, respectively). Bogart et al looked at the receipt of highly active antiretroviral treatment and physician monitoring in a small sample of 154 people with serious mental illness infected with HIV. ${ }^{71}$ Highly active antiretroviral treatment use, patterns of CD4 count and viral load monitoring did not differ significantly between individuals with both serious mental illness and HIV, and individuals with HIV only. Palepu et al looked at 231 people co-infected with HIV and hepatitis C. Of these, about half had a diagnosis, or were receiving treatment for, substance misuse. ${ }^{73}$ Twenty-two per cent of participants had received liver specialty care and $89 \%$ primary care - suggesting significant unmet need. When available, medical care was delivered by specialists in liver disease or hepatitis. There was a non-significant positive trend between substance misuse treatment and the receipt of liver speciality care, but no link with depressive symptoms. However, in those co-infected participants abstinent from alcohol for 30 days, there was a significant association with increased care. Unfortunately the only marker of care was out-patient visits in the previous 6 months and therefore results may simply reflect differences in healthcare utilisation.

Himelhoch et al found that HIV-positive individuals with either drug misuse problems or serious mental illness were more likely to receive in-patient medical care but less likely to receive highly active antiretroviral treatment medication, even after adjustment for illness severity. ${ }^{74}$ Fremont and colleagues examined inequalities in health outcomes, access and patient experience in 295 HIV-positive individuals with and without serious mental illness. ${ }^{75}$ After adjustments for multiple factors poorer medical care was present in each domain in Los Angeles but only access to care was poorer in New York.

\section{Quality of cancer care}

Goodwin et al conducted the only study to date on mental illness and the quality of cancer care. In this retrospective cohort study using the Medicare database in the USA, the authors identified 24696 women aged 67 years and older with a diagnosis of breast cancer. ${ }^{76}$ Although women with comorbid depression $(7.8 \%$ of the sample) were more likely to have visited the doctor in the previous 2 years, they were less likely to have received definitive treatment (as defined by published consensus criteria), a difference that remained after controlling for age, ethnicity, comorbidity and hospital site. This difference equated to a 19\% increase in the odds of receiving less than definitive therapy. Women with stage two or later breast cancer were less likely to receive chemotherapy $(P<0.0001)$. The women with depression were $42 \%$ more likely to die in the 3-year follow-up period (after controlling for other factors that might affect survival) (hazard ratio 1.42, 95\% CI 1.13-1.79). Interestingly, this difference remained significant after restricting the analysis to women who received definitive treatment, implying that this difference in survival cannot be explained by differences in treatment alone.

\section{Discussion}

We conducted a thorough systematic review of 31 comparative studies that examined the quality of medical care in those with and without comorbid mental illness and/or substance misuse. We did not attempt to look at possible solutions to inequalities in care: these are discussed elsewhere. ${ }^{2,77}$ Of the studies 19 out of 27 involving mental illness and 10 out of 10 involving substance misuse revealed inequalities in the quality of medical care. Looking at each type of mental illness separately, revealed only five studies that examined depression. ${ }^{48,57,61,73,76}$ Four out of these five studies showed inequalities in medical care. This is consistent with early work showing that depression predicted discontinuation of regular medical care 1 year later. ${ }^{78}$ Only three specifically examined bipolar disorders and no significant differences in quality of care were apparent in this group. ${ }^{48,58,70}$ The remainder of the studies examined schizophrenia and related disorders or broadly defined serious mental illness.

In some of these studies the magnitude of the inequality was large (online Table DS2) with differences in care approaching $50 \%$ of the comparative standard. However, results were variable. In general, across disease states, the presence of mental illness seems to affect the quality of care. Neutral results generally occurred in studies that relied upon small samples of those with mental illness, which may be influenced by type II error. ${ }^{32,70}$ However, one notable exception was from Whyte et al who found no difference in diabetes care. ${ }^{70}$ It is notable that this study is the only one of its type conducted in UK general practice. It is of some concern that poor quality of care may be translated into poor health outcomes, including mortality. In a study of preventive care for osteoporosis, Bishop and colleagues found a low rate of receipt of osteoporosis services along with a greater number of total fractures in women with schizophrenia. ${ }^{34}$ Druss et al, ${ }^{51}$ Lawrence et $a l^{54}$ and Goodwin et $a l^{76}$ all found an excess of mortality associated with deficits in quality of care.

The influence of mental illness and substance misuse on healthcare was most thoroughly studied in cardiovascular disease and diabetes mellitus. In cardiovascular care there was strong evidence of inferior quality of care although the magnitude of the effect was variable. Of ten studies that examined the comparative quality of cardiovascular care in people with and without a mental illness, seven found definitively lower quality of care in those with mental illness, ${ }^{50-52,54,55,57,59}$ six found lower quality of care for patients with substance misuse ${ }^{50-54,59}$ but two modest sized retrospective cohort studies found no difference in levels of care in the primary mental illness cohort. ${ }^{53,56}$ One study found deficits for those with schizophrenia but not bipolar disorders and only in specific domains. ${ }^{58}$ It is likely that these last three studies were underpowered to detect potentially small differences that were found in larger studies. In diabetes, there was moderate evidence of inequalities in care, and where this existed effect sizes were generally small. Thus of eleven studies that were identified, six studies demonstrated inferior care/education for those with mental illness, ${ }^{62-66,68,69}$ and one study showed inferior care for people with substance use disorders who also had diabetes. ${ }^{60}$ 
One additional report showed differences in only one out of four markers of care ${ }^{61}$ However, five studies revealed no appreciable difference in quality of care for people with primary mental illness ${ }^{60,61,65,68,70}$ although in one report those with schizophrenia were significantly more likely to receive older drugs. ${ }^{68}$

In the field of HIV and hepatitis care we found much variability in medical care. For example in one study only $54.2 \%$ of people positive for hepatitis $\mathrm{C}$ and with severe mental illness had a regular source of medical care. ${ }^{79}$ Being younger, African American, male and single were all independently associated with a lower likelihood of having a regular source of medical care. From our data no inequality was apparent in three of four studies, all of which were underpowered (online Table DS1). In the largest study individuals with HIV and either mental illness or substance misuse were underserved in terms of treatment with highly active antiretroviral medication. ${ }^{74}$ Finally, in cancer care the only study to date $^{76}$ revealed lower levels of care, despite higher rates of medical visits (see below).

Rarely did people with mental illness receive higher-quality care, although there were instances where the likelihood of obtaining a physical examination, ${ }^{49}$ the likelihood of individuals with diabetes with hypercholesterolaemia receiving a lipid-lowering agent and referral for nutritional consultation ${ }^{68}$ and the likelihood of obtaining smoking cessation counselling ${ }^{69}$ were better than expected. All such results, except smoking cessation, conflicted with other studies in this dataset showing inferior care in these domains.

\section{Explanations for inequalities in care}

There are many possible reasons for these apparent deficits in physical care for people with mental illness. ${ }^{80}$ Possibilities include competing demands for mental and physical diagnoses and interventions, other provider factors, patient barriers and systems issues. ${ }^{81,82}$ It is often thought that, because of medical and psychiatric comorbidity, seemingly unrelated conditions compete for clinicians' attention. ${ }^{83}$ Against this, recent studies suggest that the adequacy of medical care may not be adversely influenced by the number of comorbid medical disorders. ${ }^{84,85}$ Further, when types of comorbidity are compared, those with specific medical comorbidity (such as asthma) appear to be half as likely to have blood pressure or cholesterol measured in primary care than those with psychiatric comorbidity (schizophrenia). ${ }^{43}$

Among people with a psychiatric illness, help-seeking, adherence to medical treatment and attendance at follow-up appointments influence received care. ${ }^{86,87}$ The relationship between mental illness and other demographic factors may also be important. In an epidemiological survey of the Dutch population comorbid substance use, substance dependence was a predictor of lower receipt of primary care among people with depression. ${ }^{88}$ In those with severe mental illness at risk of hepatitis $\mathrm{C}$, one group found that ethnicity (being Black), gender (male) or living in a community with high exposure to community violence lowered the odds of receiving appropriate care. ${ }^{89}$ At the same time, clinician factors such as willingness to investigate, ability and enthusiasm to treat and willingness to offer follow-up are important predictors of quality of care. In a large health survey of veteran participants Kilbourne et al reported that people with comorbid serious mental illness were less likely to be told by providers that they had chronic medical conditions, suggesting underrecognition of medical illness in this group. ${ }^{90}$ Similarly, patients primarily cite barriers to care involving availability of medical advice and quality of medical advice. ${ }^{91-93}$ Indirect evidence suggests that clinicians' attitudes towards patients directly influence health outcomes. In one study in primary care, poor mental health status was linked with poor accessibility, poorer general practitioner attitude and less time spent with the general practitioner. $^{94}$ In a study of 59 people seen in a US community mental health centre, $14 \%$ reported that they used the medical emergency department for their routine medical care needs, $45 \%$ said that their mental health provider did not ask them about medical issues and 39\% said that their medical provider did not ask about mental health issues. ${ }^{91}$

Further, the type of mental illness may be important in attendance and adherence to care, as shown in the findings that quality of care may be influenced by the presence of substance misuse. ${ }^{95}$ People living in the community who are depressed or distressed seek help more often and use more general healthcare resources than those who are not depressed or distressed. ${ }^{96}$ Depression is associated with an excess of medical visits even after adjusting for the number of coexisting medical disorders. ${ }^{9}$ Less certain however is the healthcare utilisation of medical outpatients who have psychiatric comorbidity and in particular the healthcare utilisation of those with serious mental illness. In this dataset healthcare utilisation was measured in two studies set in general (internal) medicine. One found lower frequency of medical visits ${ }^{48}$ whereas the other suggested that people with mental illness were more likely to visit a doctor. ${ }^{49}$ Only one study examined healthcare utilisation in cardiovascular settings and found low rates of hospitalisation in people with dementia and schizophrenia compared with controls. In diabetes, one study showed lower rates of emergency readmission, ${ }^{66}$ another higher rates of attendance ${ }^{60}$ but two found no differences in attendance for people with mental illness. ${ }^{62,64}$ Importantly, people may attend less often for preventive and routine care but be forced to attend more frequently for emergency care. ${ }^{97,98}$ For example, Desai et al, ${ }^{60}$ Jones et $a l^{56}$ and Goodwin et $a l^{76}$ all found that individuals with medical illness and substance use disorders or other mental illness had higher rates of healthcare utilisation compared with those with medical illness alone. Yet all three studies showed that despite higher attendance, people with comorbidity were less likely to have received definitive treatment. Krein and colleagues found no better care in those with mental illness despite a higher rate of attendance. ${ }^{65}$ Similarly Salsberry et al found that compared with the general population, women with serious mental illness had more emergency department visits, visited a doctor more frequently but, despite this high healthcare utilisation, had very low rates of cervical smears and mammograms. ${ }^{30}$ Of note is the fact that they also showed that individuals with serious mental illness were less likely to have an appointment for general internal medicine in contrast to generally higher rates of visits, suggesting that visits targeted at delivering medical care may be deficient, even in the face of frequent attendance in primary care or emergency departments. Kaplowitz and colleagues stratified individuals by attendance, finding that those with psychiatric diagnoses received lower levels of care, but only in those who infrequently used out-patient services and this was reversed in those with more out-patient visits. ${ }^{31}$ Therefore, although people with mental illness may have more frequent medical contact, it may be ineffective and the quality of care offered to individuals may be unsatisfactory in different medical settings. Thus frequency of attendance (uptake of care) appears to be largely independent of inferior quality of received care and therefore this factor cannot be supported as the main explanatory variable.

\section{Limitations}

This review has several limitations, most notably the small sample sizes in some areas of medical care, heterogeneity and the inherent 
limitations of administrative data in most large-scale studies. In particular most of the adequately powered studies included only minimal data on the nature of the medical case itself and generally lacked robust methods of establishing psychiatric diagnoses. Additionally outcome measures were diverse and difficult to unite quantitatively. As a result more sophisticated meta-analysis could not be conducted and a full explanation for inequalities in care was not possible. In addition we were unable to examine the effect of care systems, although the well-powered negative finding from Whyte et $a l^{70}$ in relation to UK primary care suggests that this would be a valuable area for further study.

\section{Summary}

There is strong evidence to support inequalities in medical care disadvantaging those who have a psychiatric illness or a substance use disorder. Despite promising approaches to shared care there is a substantial gap in routine medical care for many individuals with mental illness or substance use disorders. ${ }^{2,99,100}$ This is most apparent in general (internal) medicine and cardiovascular care but may also be present in diabetes care and cancer care. There is little evidence to suggest that the recommended enhanced medical care for individuals with mental illness has been successfully implemented. Future work must focus on the type and severity of mental illness, patient factors such as adherence and systems interventions to increase the quality of care for those with chronic mental illness.

Alex J. Mitchell, MRCPsych, Department of Liaison Psychiatry, Leicester Genera Hospital, and Department of Cancer and Molecular Medicine, Leicester Royal Infirmary, Leicester, UK, Darren Malone, MRCPsych, Lakes District Health Board New Zealand, and Department of Health Science, Leicester University, UK; Caroline Carney Doebbeling, MD, MSC, Department of Internal Medicine, Indiana University school of Medicine, Regenstrief Institute, Indianapolis, USA

Correspondence: Alex J. Mitchell, Department of Liaison Psychiatry, Leicester General Hospital, Leicester LE5 4PW, UK. Email: Alex.mitchel|@Ieicspart.nhs.uk

First received 26 Sep 2007, final revision 30 Sep 2008, accepted 20 Nov 2008

\section{Appendix}

\section{Criteria for critical appraisal}

\section{Methodological area and standard} Study design

cross-sectional

Prospective or cohort

Sample quality

0-99

$>100$

$>200$

$>1000$

$>10000$

$>100000$

Adequacy of comparators

Unmatched comparison sample

Equivalent comparison sample

Matched comparison sample (near identical)

Consideration of confounders

None

Demographics

(As above) plus medical or social factors

(As above) plus healthcare factors

(As above) plus additional factors

Adequacy of outcome measure

No outcome measures

Simple monitoring alone

Collection of disease variables

Extensive examination of impact
Separation of delivery and uptake of care

No adjustment

Partial adjustment

Measured but not adjusted

Full adjustment

Overall methodological score: 0-3 poor; 4-7 below average;

8-11 adequate; 12-15 good; 16-20 excellent.

\section{References}

1 US Department of Health and Human Services. Mental Health: A Report of the Surgeon General - Executive Summary. US Department of Health and Human Services, Substance Abuse and Mental Health Services

Administration, Center for Mental Health Services, National Institutes of Health, National Institute of Mental Health, 1999.

2 Pincus HA, Page AEK, Druss B, Appelbaum PS, Gottlieb G, England MJ. Can psychiatry cross the quality chasm? Improving the quality of health care for mental and substance use conditions. Am J Psychiatry 2007; 164: 712-9.

3 Lyketsos CG, Dunn G, Kaminsky MJ, Breakey WR. Medical comorbidity in psychiatric inpatients: relation to clinical outcomes and hospital length of stay. Psychosomatics 2002; 43: 24-30.

4 Kessler RC, Ormel J, Demler O, Stang PE. Comorbid mental disorders account for the role impairment of commonly occurring chronic physical disorders: results from the national comorbidity survey. J Occup Environ Med 2003; 45: 1257-66.

5 Warner LA. Medical problems among adolescents in US mental health services: relationship to functional impairment. J Behav Health Serv Res 2006; 33: 366-79.

6 Scott KM, McGee MA, Wells JE, Browne MAO. Disability in Te Rau Hinengaro: the New Zealand Mental Health Survey. Aust N Z J Psychiatry 2006; 40: 889-95.

7 Unützer J, Patrick DL, Diehr P, Simon G, Grembowski D, Katon W. Quality adjusted life years in older adults with depressive symptoms and chronic medical disorders. Int Psychogeriatrics 2000; 12: 15-33.

8 Baumeister H, Balke K, Harter M. Psychiatric and somatic comorbidities are negatively associated with quality of life in physically ill patients. J Clin Epidemiol 2005; 58: 1090-100

9 Baune BT, Adrian I, Jacobi F. Medical disorders affect health outcome and general functioning depending on comorbid major depression in the general population. J Psychosom Res 2007; 62: 109-18.

10 Felker B, Yazel JJ, Short D. Mortality and medical comorbidity among psychiatric patients. A review. Psychiatr Serv 1996; 47: 1356-63.

11 Wells KB, Rogers W, Burnam A, Greenfield S, Ware Jr JE. How the medical comorbidity of depressed patients differs across health care settings: results from the Medical Outcomes Study. Am J Psychiatry 1991; 148: 1688-96.

12 Koranyi EK, Potoczny WM. Physical illnesses underlying psychiatric symptoms. Psychother Psychosom 1992; 58: 155-60.

13 Singleton N, Bumpstead R, O'Brien M, Lee A, Meltzer H. Psychiatric morbidity among adults living in private households, 2000. Int Rev Psychiatry 2003; 15: 65-73.

14 Yates WR, Mitchell J, Rush AJ, Trivedi MH, Wisniewski SR, Warden D, et al. Clinical features of depressed outpatients with and without co-occurring general medical conditions in STAR*D. Gen Hosp Psychiatry 2004; 26: 421-9.

15 Aragones E, Pinol JL, Labad A. Depression and physical comorbidity in primary care. J Psychosom Res 2007; 63: 107-11.

16 Alaja R, Seppa K, Sillanaukee P, Tienari P, Huyse FJ, Herzog T, et al. Physical and mental comorbidity of substance use disorders in psychiatric consultations. Alcoholism: Clin Exp Res 1998; 22: 1820-4.

17 Mertens JR, Lu YW, Parthasarathy S, Moore C, Weisner CM. Medical and psychiatric conditions of alcohol and drug treatment patients in an $\mathrm{HMO}$ comparison with matched controls. Arch Intern Med 2003; 163: 2511-7.

18 De Alb I, Samet JH, Saitz R. Burden of medical illness in drug- and alcoholdependent persons without primary care. Am J Addict 2004; 13: 33-45.

19 Mertens JR, Flisher AJ, Fleming MF, Weisner CM. Medical conditions of adolescents in alcohol and drug treatment. Comparison with matched controls. J Adolesc Health 2007; 40: 173-9.

20 Department of Health. National Service Framework for Mental Health. Modern Standards and Service Models. Stationery Office, 1999.

21 Unützer J, Schoenbaum, M, Druss BG, Katon WJ. Transforming mental health care at the interface with general medicine: report for the Presidents Commission. Psychiatr Serv 2006; 57: 37-47.

22 Campbell SM, Roland MO, Buetow SA. Defining quality of care. Soc Sci Med 2000; 51: 1611-25. 
23 Desai MM, Rosenheck RA. Unmet need for medical care among homeless adults with serious mental illness. Gen Hosp Psychiatry 2005; 27: 418-25.

24 Lohr KN (ed). Medicare: A Strategy for Quality Assurance, Vol. 1. National Academy Press, 1990.

25 National Quality Measures Clearinghouse. What is a Quality Measure? (http:// www.qualitymeasures.ahrq.gov/resources/measure_use.aspx).

26 Moher D, Cook DJ, Eastwood S, Olkin I, Rennie D, Stroup DF. Improving the quality of reports of meta-analyses of randomised controlled trials: the QUOROM statement. Quality of Reporting of Meta-analyses. Lancet 1999; 354: $1896-900$.

27 Oxford Centre for Evidence-Based Medicine. Guidance for Critical Appraisal (http://www.cebm.net/critical_appraisal.asp).

28 Craig JC, Irwig LM, Stockler MR. Evidence-based medicine: useful tools for decision making. MJA 2001; 174: 248-53 (http://www.mja.com.au/public/ issues/174_05_050301/craig/craig.html).

29 Druss BG, Rosenheck RA, Desai MM, Perlin JB. Quality of preventative care for patients with mental disorders. Medical Care 2002; 40: 129-36.

30 Salsberry PJ, Chipps E, Kennedy C. Use of general medical services among Medicaid patients with severe and persistent mental illness. Psychiatr Serv 2005; 56: 458-62.

31 Kaplowitz RA, Scranton RE, Gagnon DR, Cantillon C, Cantillon C, Levenson JW, et al. Health care utilization and receipt of cholesterol testing by veterans with and those without mental illness. Gen Hosp Psychiatry 2006; 28 : 137-44

32 Steiner J, Hoff RA, Moffett C, Reynolds H, Mitchell M, Rosenheck R, et al. Preventative health care for mentally ill women. Psychiatr Serv 1998; 49: 696-8.

33 Lindamer LA, Buse DC, Auslander L. A comparison of gynecological variables and service use among older women with and without schizophrenia. Psychiatr Serv 2003; 54: 902-4.

34 Bishop JR, Alexander B, Lund BC, Klepser TB. Osteoporosis screening and treatment in women with schizophrenia: a controlled study. Pharmacotherapy 2004; 24; 515-21.

35 Dolder CR, Furte K, Lacro JP, Jeste DV. Antihypertensive medication adherence and blood pressure control in patients with psychotic disorders compared to persons without psychiatric illness. Psychosomatics 2005; 46 135-41.

36 Trief PM, Morin PC, Izquierdo R, Teresi J, Eimicke JP, Goland R, et al. Depression and glycemic control in elderly ethnically diverse patients with diabetes. The IDEATel Project. Diabetes Care 2006; 29: 830-5.

37 Gross R, Olfson M, Gameroff MJ, Carasquillo O, Shea S, Feder A, et al. Depression and glycemic control in hispanic primary care patients with diabetes. J Gen Intern Med 2005; 20: 460-6.

38 Lustman PJ, Clouse RE. Depression in diabetic patients: the relationship between mood and glycemic control. J Diabetes Complications 2005; 19 113-22.

39 Murdoch RO, Chelminski P, Malone RM, Dewalt DA, Pignone M. Depression and diabetes: does treating depression improve glycemic control in patients with diabetes. Systematic review and meta-analysis. J Gen Intern Med 2005; 20 (suppl 1): 63

40 Daumit GL, Pronovost PJ, Anthony CB, Guallar, E, Steinwachs DM, Ford DE. Adverse events during medical and surgical hospitalizations for persons with schizophrenia. Arch Gen Psychiatry 2006; 63: 267-72.

41 Nasrallah HA. Low rates of treatment for metabolic disorders in the CATIE schizophrenia trial at baseline. Neuropsychopharmacology 2005; 30 (suppl 1): $\mathrm{S} 204$.

42 Folsom DP, McCahill M, Bartels SJ, Lindamer LA, Ganiats TG, Jeste DV, et al: Medical comorbidity and receipt of medical care by older homeless people with schizophrenia or depression. Psychiatr Serv 2002; 53: 1456-60.

43 Rifai MA, Moles JK, Short DD. Hepatitis C teatment eigibility and outcomes among patients with pychiatric ilness. Psychiatr Serv 2006; 57: 570-2.

44 Roberts L, Roalfe A, Wilson S, Lester H. Physical health care of patients with schizophrenia in primary care: a comparative study. Fam Pract 2007; 24 34-40.

45 Hwang SS, Chang VT, Cogswell J, Alejandro Y, Osenenko P, Morales E, et al Study of unmet needs in symptomatic veterans with advanced cancer: incidence, independent predictors and unmet needs outcome model. J Pain Symptom Manage 2004; 28: 421-32.

46 Redelmeier D, Tan SH, Booth GL. The treatment of unrelated disorders in patients with chronic medical diseases. N Eng J Med 1998; 338: 1516-20.

47 Desai M, Rosenheck RA, Druss BG, Perlin JB. Receipt of nutrition and exercise counselling among medical outpatients with psychiatric and substance use disorders. J Gen Intern Med 2002; 17: 556-60.
48 Cradock-O'Leary J, Young AS, Yano EM, Wang M, Lee ML. Use of general medical services by VA patients with psychiatric disorders. Psychiatr Serv 2002; 53: 874-8.

49 Dickerson F, McNary SW, Brown CH, Kreyenbuhl J, Goldberg RW, Dixon LB. Somatic healthcare utilization among adults with serious mental illness who are receiving community psychiatric services. Med Care 2003; 41: 560-70.

50 Druss B, Bradford DW, Rosenheck RA, Radford MJ, Krumholz HM. Mental disorders and use of cardiovascular procedures after myocardial infarction. JAMA 2000; 283: 506-11.

51 Druss, BG, Bradford D, Rosenheck RA, Radford MJ, Krumholz HM. Quality of medical care and excess mortality in older patients with mental disorders. Arch Gen Psychiatry 2001; 58: 565-72.

52 Young J, Foster D. Cardiovascular procedures in patients with mental disorders. JAMA 2000; 28: 3198-9.

53 Desai MM, Rosenheck RA, Druss BG, Perlin JB. Mental disorders and quality of care among postacute myocardial infarction outpatients. J Nerv Ment Dis 2002; 190: 51-3.

54 Lawrence DM, Holman CDJ, Jablensky AV, Hobbs MST. Death rate from ischaemic heart disease in Western Australian psychiatric patients 19801998. Br J Psychiatry 2003; 182: 31-6.

55 Petersen LA, Normand SL, Druss BG, Rosenheck RA. Process of care and outcome after acute myocardial infarction for patients with mental illness in the VA health care system: are there disparities? Health Serv Res 2003; 38: 41-63.

56 Jones LE, Carney CP. Mental disorders and revascularization procedures in a commercially insured sample. Psychosom Med 2005; 67: 568-76.

57 Wang P, Avorn J, Brookhart MA, Mogun H, Schneeweiss S, Fischer MA, et al. Effects of noncardiovascular comorbidities on antihypertensive use in elderly hypertensives. Hypertension 2005; 46: 273-9.

58 Hippisley-Cox J, Parker C, Coupland C, Vinogradova Y. Inequalities in the primary care of patients with coronary heart disease and serious mental health problems: a cross-sectional study. Heart 2007; 93: 1256-62.

59 Li Y, Glance LG, Cai X, Mukamael DB. Are patients with co-existing mental disorders more likely to receive CABG surgery from low-quality cardiac surgeons? The experience in New York State. Med Care 2007; 45: 587-93.

60 Desai M, Rosenheck RA, Druss BG, Perlin JB. Mental disorders and quality of diabetes care in the veterans health administration. Am J Psych 2002; 159: 1584-90.

61 Lin EH, Katon W, Von Korff M, Rutter C, Simon GE, Oliver M, et al. Relationship of depression and diabetes self-care, medication adherence, and preventive care. Diabetes Care 2004; 27: 2154-60.

62 Dixon L. A comparison of type II Diabetes among persons with and without severe mental illness. Psychiatr Serv 2004; 55: 892-900.

63 Jones L, Clarke W, Carney CP. Receipt of diabetic services by insured adults with and without claims for mental disorders. Med Care 2004; 42: 1167-75.

64 Frayne SM, Halanych JH, Miller DR, Wang F, Lin H, Pogach L, et al. Disparities in diabetes care: impact of mental illness. Arch Int Med 2005 165: 2631-8.

65 Krein SL, Bingham R, McCarthy JF, Mitchinson A, Payes J, Valenstein, M. Diabetes treatment among VA patients with comorbid serious mental illness. Psychiatr Serv 2006; 57: 1016-21.

66 Kreyenbuhl J, Dickerson F, Medoff D, Brown CH, Goldberg RW, Fang L, et al. Extent and management of cardiovascular risk factors in patients with type 2 diabetes and serious mental illness. J Nerv Ment Dis 2006; 194: 404-10.

67 Sullivan G, Han X, Moore S, Kotrla K. Disparities in hospitalization for diabetes among persons with and without co-occurring mental disorders. Psychiatr Serv 2006; 57: 1126-31.

68 Weiss AP, Henderson DC, Weilburg JB, Goff DC, Meigs JB, Cagliero E, et al. Treatment of cardiac risk factors among patients with schizophrenia and diabetes. Psychiatr Serv 2006; 57: 1145-52.

69 Goldberg RW, Kreyenbuhl JA, Medoff DR, Dickerson FB, Wohlheiter K, Fang $\sqcup$, et al. Quality of diabetes care among adults with serious mental illness. Psychiatr Serv 2007; 58: 536-43.

70 Whyte S, Penny C, Phelan M, Hippisley-Cox J, Majeed A. Quality of diabetes care in patients with schizophrenia and bipolar disorder: cross-sectional study. Diabet Med 2007; 24: 1442-8.

71 Butt AA, Wagener M, Shakil AO, Ahmed J. Reasons for non-treatment of hepatitis $C$ in veterans in care. J Viral Hepatitis 2005; 12: 81-5.

72 Bogart LM, Fremont AM, Young AS, Pantoja P, Chinman M, Morton S, et al. Patterns of HIV care for patients with serious mental illness. AIDS Patient Care STD 2006; 20: 175-82.

73 Palepu A, Cheng DM, Kim T, Nunes D, Vidaver J, Alperen J, et al. Substance abuse treatment and receipt of liver specialty care among persons coinfected with HIV/HCV who have alcohol problems. I Subst Abuse Treat 2006; 31: 411-7. 
74 Himelhoch S, Chander G, Fleishman JA, Hellinger J, Gaist P, Gebo KA. Access to HAART and utilization of inpatient medical hospital services among HIVinfected patients with co-occurring serious mental illness and injection drug use. Gen Hosp Psychiatry 2007; 29: 518-25.

75 Fremont AM, Young AS, Chinman M. Differences in HIV care between patients with and without severe mental illness. Psychiatr Serv 2007; 58 681-8.

76 Goodwin JS, Zhang DD, Ostir GV. Effect of depression on diagnosis, treatment, and survival of older women with breast cancer. J Am Geriatr Soc 2004; 52: 106-11.

77 Institute of Medicine Committee on Quality of Health in America. Improving the Quality of Health Care for Mental and Substance Use Conditions: 77-139. National Academies Press, 2006.

78 Cooper-Patrick L, Crum RM, Pratt LA, Eaton WW, Ford DE. The psychiatric profile of patients with chronic diseases who do not receive regular medica care. Int J Psychiatry Med 1999; 29: 165-80.

79 Swartz MS, Swanson JW, Hannon MJ, Bosworth HS, Osher FC, Essock SM, et al. Blood-borne infections and persons with mental illness: regular sources of medical care among persons with severe mental illness at risk of hepatitis C infection. Psychiatr Serv 2003; 54: 854-9.

80 Kim MM, Swanson JW, Swartz MS, Bradford DW, Mustillo SA, Elbogen EB. Healthcare barriers among severely mentally ill homeless adults: evidence from the five-site health and risk study. Admin Policy Ment Health 2007; 34 363-75

81 Palmer RH. Process-based measures of quality: the need for detailed clinica data in large health care databases. Ann Intern Med 1997; 127: 733-8.

82 Campbell SM, Braspenning J, Hutchinson A, Marshall M. Research methods used in developing and applying quality indicators in primary care. Qual Saf Health Care 2002; 11: 358-64.

83 Piette JD, Kerr EA. The impact of comorbid chronic conditions on diabetes care. Diabetes Care 2006; 29: 725-31.

84 Min LC, Wenger NS, Fung C, Chang JT, Ganz DA, Higashi T, et al. Multimorbidity is associated with better quality of care among vulnerable elders. Med Care 2007; 45: 480-8.

85 Higashi T, Wenger NS, Adams JL, Fung C, Roland M, McGlynn EA, et al. Relationship between number of medical conditions and quality of care. $N$ Eng J Med 2007; 356: 2496-504.

86 Kerr EA, Krein SL, Vijan S, Hofer TP, Hayward RA. Avoiding pitfalls in chronic disease quality measurement: a case for the next generation of technical quality measures. Am J Manag Care 2001; 7: 1033-43.

87 Powell $A E$, Davies HTO, Thomson RG. Using routine comparative data to assess the quality of health care: understanding and avoiding common pitfalls. Qual Saf Health Care 2003; 12: 122-8.
88 ten Have M, de Graaf R, Vollebergh W, Beekman A. What depressive symptoms are associated with the use of care services? Results from the Netherlands Mental Health Survey and Incidence Study (NEMESIS). J Affect Disord 2004; 80: 239-48.

89 Swartz MS, Swanson JW, Hannon MJ, Bosworth HS, Osher FC, Essock SM, et al. Regular sources of medical care among persons with severe mental illness at risk of hepatitis C infection. Psychiatr Serv 2003; 54: 854-9.

90 Kilbourne AM, McCarthy JF, Welsh D, Blow F. Recognition of co-occurring medical conditions among patients with serious mental illness. J Nerv Ment Dis 2006; 194: 598-602.

91 Druss BG, Rosenheck RA. Mental disorders and access to medical care in the United States. Am J Psychiatry 1998; 155: 1775-7.

92 Levinson Miller C, Druss BG, Dombrowski EA, Rosenheck RA. Barriers to primary medical care among patients at a community mental health center. Psychiatr Serv 2003; 54: 1158-60.

93 O'Day B, Killeen MB, Sutton J, lezzoni LI. Primary care experiences of people with psychiatric disabilities: barriers to care and potential solutions Psychiatr Rehabil J 2005; 28: 339-45.

94 Al-Mandhari AS, Hassan AA, Haran D. Association between perceived health status and satisfaction with quality of care: evidence from users of primary health care in Oman. Fam Pract 2004; 21: 519-27.

95 Inagaki T, Yasukawa R, Okazaki S, Yasuda H, Kawamukai T, Utani E, et al Factors disturbing treatment for cancer in patients with schizophrenia. Psychiatry Clin Neurosci 2006; 60: 327-31.

96 Stein MB, Cox BJ, Afifi TO, Belik SL, Sareen J. Does co-morbid depressive illness magnify the impact of chronic physical illness? A population-based perspective. Psychol Med 2006; 36: 587-96.

97 Berren MR, Santiago JM, Zent MR, Carbone CP. Health care utilization by persons with severe and persistent mental illness. Psychiatr Serv 1999; 50 559-61.

98 Hackman AL, Goldberg RW, Brown CH, Fang LJ, Dickerson FB, Wohlheiter K, et al. Use of emergency department services for somatic reasons by people with serious mental illness. Psychiatr Serv 2006; 57: 563-6.

99 Weisner C, Mertens J, Parthasarathy S, Moore C, Lu Y. Integrating primary medical care with addiction treatment: a randomized controlled trial. JAMA 2001; 286: 1715-23.

100 Knott A, Dieperink E, Willenbring ML, Heit S, Durfee JM, Wingert M, et al. Integrated psychiatric/medical care in a chronic hepatitis $C$ clinic: effect on antiviral treatment evaluation and outcomes. Am J Gastroenterol 2006; 101 2254-62.

(1) 